\title{
Measuring the thermal resistance of vertical interfaces separating two different media using lock-in infrared thermography with laser spot excitation
}

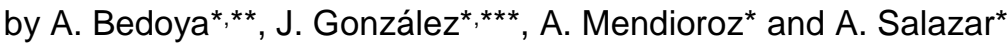

\begin{abstract}
* Departamento de Física Aplicada I, Escuela de Ingeniería de Bilbao, Universidad del País Vasco UPV/EHU, Plaza Ingeniero Torres Quevedo 1, 48013 Bilbao, Spain, agustin.salazar@ehu.es

**Instituto Politécnico Nacional (IPN), Centro de Investigación en Ciencia Aplicada y Tecnología Avanzada (CICATA), Unidad Legaria, Legaria 694, Col. Irrigación, C.P. 11500, Ciudad de México, Mexico, adrian_filipo@hotmail.com

***Department of Applied Physics, CINVESTAV Unidad Mérida, carretera Antigua a Progreso km6, A.P. 73 Cordemex, Mérida Yucatán 97310, Mexico, jorge.gonzalez860824@hotmail.com
\end{abstract}

\begin{abstract}
We propose a method to measure the thermal contact resistance at the interface between two different media using lock-in infrared thermography with laser spot heating. We find an analytical expression for the surface temperature of the two-media when they are illuminated by a modulated Gaussian laser spot. The surface temperature on the twomedia with calibrated interface thickness is measured using an infrared camera. By fitting the surface temperature to the model, the thermal resistance of the interface is obtained. A good agreement between the nominal and retrieved thickness of the interface is found confirming the validity of the model.
\end{abstract}

\section{Introduction}

The growing necessity of in-service non-destructive testing and evaluation of surface breaking cracks in a wide variety of devices has been a challenging task for modern industries and laboratories. Several well-established methodologies like dye penetrants, magnetic particles, eddy currents, and x-rays have been used to detect fissures as well as defects in materials. In the last decades, infrared thermography (ultrasonically, inductively and optically excited) has been applied to detect this kind of defects.

In a recent work, the authors sized the thermal resistance of infinite vertical cracks using lock-in infrared thermography, by illuminating the sample surface with a modulated and focused laser spot close to the crack [1]. In this work, we extend the previous method to characterize the thermal contact resistance at the interface between two different media. This method could be useful to characterize the junction between two solids (e.g. welding) or in the quality control of contacts between different parts of composite materials.

\section{Theory}

Fig. 1 shows the cross-section showing the interface between two opaque media 1 and 2 . The interface is placed at plane $y=0$. The surface of medium 2 is illuminated by a laser beam of power $P_{o}$, modulated at a frequency $f(\omega=2 \pi f)$. The center of the Gaussian laser spot is located at a distance $d$ from the interface and its radius is $a$ (at $1 / \mathrm{e}^{2}$ of the intensity). Adiabatic boundary conditions at the sample surface are assumed.

The heat diffusion equation in each medium has been solved in the Hankel space, using the images method. The temperature at any point in the two-media contains a triple integral and is quite time consuming. However, the temperature profile along the $y$-axis reduces to a semi-analytical expression containing a single integral, which is very fast to calculate:

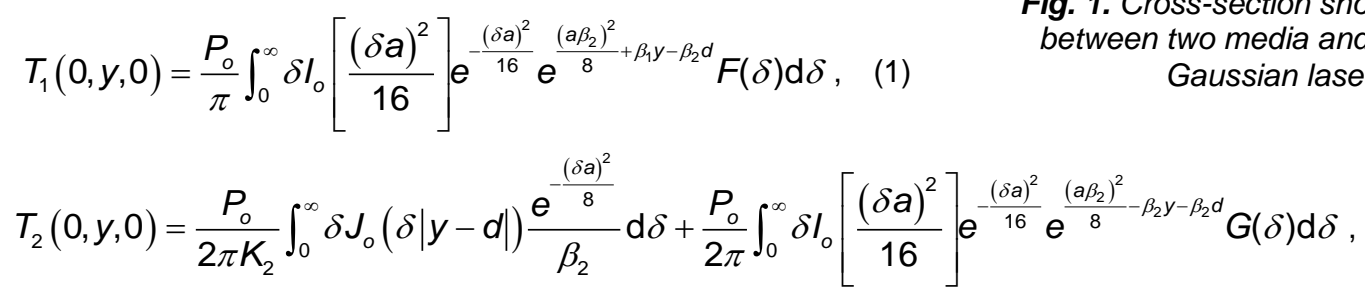

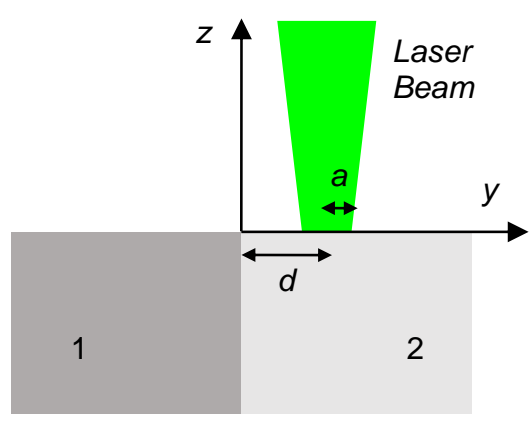

Fig. 1. Cross-section showing the interface between two media and illuminated by a Gaussian laser beam. 


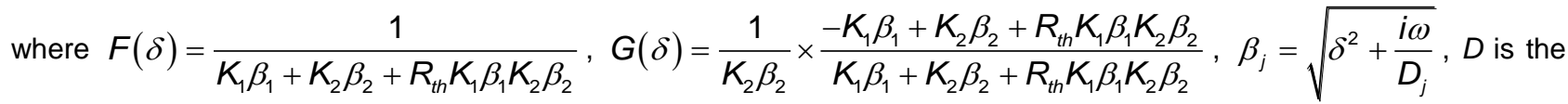
thermal diffusivity, $K$ is thermal conductivity, $J_{0}$ and $I_{0}$ are the Bessel function and the modified Bessel function of the first kind respectively. Subscripts 1 and 2 refer to medium 1 and 2 respectively. $R_{t h}=L_{\text {interface }} / K_{\text {air }}$ is the thermal contact resistance, related to the interface opening (Linterface). According to factors $F(\delta)$ and $G(\delta)$, the thermal resistance is correlated to the thermal conductivities of both media through the factor $R_{t h} K_{1} K_{2}$. This means that tightly closed interfaces between high thermal conducting materials (metals, alloys, ceramics...) are better detected than between thermal insulators (polymers, composites...).

\section{Experimental results and discussion}

We have prepared calibrated vertical interfaces by putting in contact one block of AISI-304 stainless steel and a block of PEEK polymer (Polyether-ether-ketone). A thin graphite layer about $3 \mu \mathrm{m}$ thick has been deposited onto the surface to increase both the absorption of the heating laser and the emissivity at infrared wavelengths. A wedge air gap between them has been obtained by putting a $50 \mu \mathrm{m}$ thick nickel tape at one extreme between them. In this way, we simulate an air gap of continuously varying thickness between 0 and $50 \mu \mathrm{m}$. In Fig. 2a we show the temperature amplitude thermogram at $0.42 \mathrm{~Hz}$ when a laser spot of $170 \mathrm{~mW}$ heats the stainless steel block. In the lock-in process, by averaging $10^{4}$ images (about 1 minute), the noise level is reduced below $1 \mathrm{mK}$. As can be seen, the interface between the blocks is clearly detected. Fig. $2 b$ shows three profiles of the natural logarithm of the temperature amplitude, $\operatorname{Ln}(I T)$, across the wedge, corresponding to air gaps of 50, 30 and $20 \mu \mathrm{m}$, which were controlled by an optical microscope. The $\operatorname{Ln}(I T)$ values are shifted in order to better show the temperature jump at the crack position. Dots represent the experimental data and the continuous lines the fit to Eqs. (1) and (2). A non-linear least square fit based on the Levenberg-Marquardt algorithm was implemented with three unknown parameters ( $R_{t h}, a$ and $d$ ). Anyway, as the values of $a$ and $d$ were estimated independently, in the fitting procedure they are bounded within reasonable limits around the estimated ones. The obtained air gaps $L_{\text {interface }}=50 \pm 4,34 \pm 3$ and $24 \pm 2 \mu \mathrm{m}$ are in good agreement with the optically estimated width values.
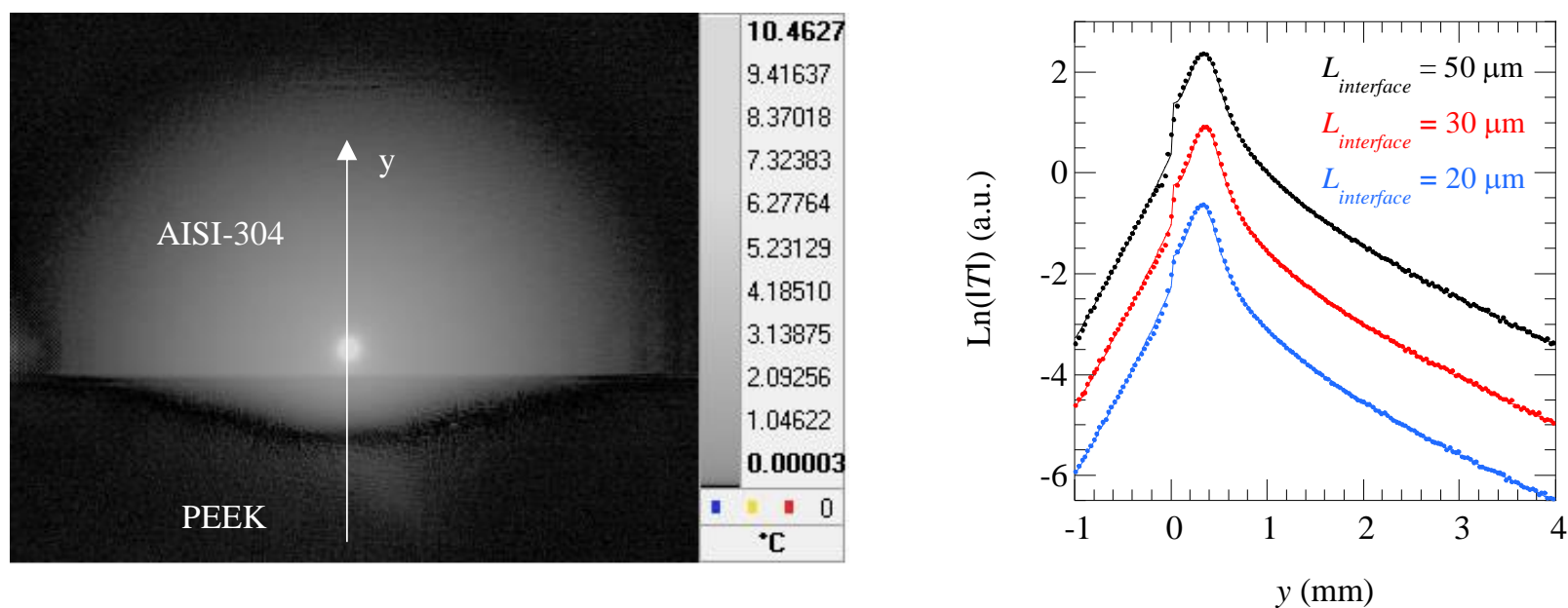

Fig. 2. (a) Temperature amplitude thermogram at $0.42 \mathrm{~Hz}$ for an AISI-304 block in contact with a PEEK block. Between them there is a wedge air gap between 0 and $50 \mu \mathrm{m}$. (b) $L n(1 \mathrm{~T})$ profiles across the wedge. Dots correspond to experimental data and continuous lines to the fit to Eqs. (1) and (2)

This work has been supported by Ministerio de Economía y Competitividad, (DPI2016-77719-R, AEI/FEDER, UE), by Universidad del País Vasco UPV/EHU (GIU16/33) and by Conacyt (Beca Mixta 2017 Movilidad en el extranjero).

\section{REFERENCES}

[1] Pech-May N. W., Oleaga A., Mendioroz A., Omella A. J., Celorrio R. and Salazar A., "Vertical cracks characterization using lock-in thermography: I. Infinite cracks". Meas. Sci. Technol., 25, 115601(10pp), 2014. 